\title{
Editorial
}

\section{ARDS and sepsis - definitions and new therapy}

The term adult respiratory distress syndrome (ARDS) is used to describe a group of disorders that cause acute lung injury. However, as a result of differences in terminology, the definition of ARDS lacked specificity and created difficulties in making the clinical diagnosis and comparing clinical investigations. ${ }^{1}$ Criteria used in the first description of ARDS $^{2}$ include tachypnoea, dyspnoea, the degree of hypoxaemia, radiographic infiltrates on chest $x$-ray and reduction in lung compliance. These criteria were expanded by Murray $e t$ al. ${ }^{\prime}$ to include the stage (acute/chronic) of the disease, the aetiology of the underlying disease process and the severity of acute lung injury, as assessed by a lung injury score. This score is calculated using the extent of the infiltrate on chest $x$ ray, the $\mathrm{PaO}_{2} / \mathrm{FlO}_{2}$ ratio, the level of positive endexpiratory pressure (PEEP) required to maintain the $\mathrm{PaO}_{2} / \mathrm{FlO}_{2}$ ratio, and respiratory system compliance. This definition was a considerable advance in establishing an accepted terminology for ARDS and with minor modifications has been used to describe ARDS in recent clinical trials. ${ }^{3-5}$

The terminology used to describe septic states has been even more variable and limiting to the interpretation of clinical investigations of sepsis. The American College of Chest Physicians/Society of Critical Care (ACCP/ SCCM) Consensus Conference (August 1991) ${ }^{6}$ emphasized that the use of varying definitions for the terms infection, bacteraemia, sepsis, septicaemia, septic syndrome and septic shock as well as the term septic syndrome for a non-infectious inflammatory state created problems comparing results from clinical trials. The Consensus Conference Committee developed a phrase, the systemic inflammatory response syndrome (SIRS), to describe a generalized inflammatory response, which can be caused by several insults including endotoxin. ${ }^{6}$ SIRS is manifested by two or more of the following: (1) tem-

From the Department of Anaesthesia, St. Michael's Hospital, University of Toronto.

Accepted for publication 27th April, 1993. perature $>38^{\circ} \mathrm{C}$ or $<36^{\circ} \mathrm{C}$; (2) heart rate $>90 \mathrm{bpm}$; (3) respiratory rate $>20 \mathrm{bpm}$ or $\mathrm{PaCO}_{2}<32 \mathrm{mmHg}$ (4.3 KPa); and (4) white blood cell count $>12 \times 10^{9} / 1$ or $<4 \times 10^{9} / 1$ or $>10 \%$ immature (band) forms. Sepsis is then defined as the systemic inflammatory response to infection as manifested by the clinical features of SIRS. A schematic diagram from the ACCP/SCCM Consensus Conference ${ }^{6}$ (Figure) illustrates how infection, bacteraemia, sepsis, and SIRS can be related. Clarifying terminology for both ARDS and sepsis is essential if we are to evaluate the outcome of new and innovative approaches to treating critically ill patients with sepsisinduced ARDS.

The pulmonary manifestations of ARDS have many common features despite a diverse group of underlying primary disease processes such as trauma, sepsis, aspiration and pancreatitis. Patient survival clearly depends on the reversibility of the underlying disease process. Treatment has remained empirical, including supportive interventions such as positive-pressure ventilation, PEEP, intravascular volume and cardiac output manipulation, as well as treatment of the primary disease process. In spite of an advanced understanding of the pathophysiology, the mortality for severe sepsis-related ARDS remains between 40 and $70 \%{ }^{7}$

In this issue of the Journal, a case is reported by Ezri et al. ${ }^{8}$ of a 72-yr-old man who developed severe respiratory distress and hypoxaemia in the immediate postoperative period. This ARDS (caused by bactaeremia and sepsis) complicated weaning from the ventilator after a radical neck dissection. Rapid reversal of hypoxaemia and the associated acute lung injury occurred with supportive management because the source of sepsis was removed rapidly. This is a common clinical experience when a reversible source of infection is detected and eradicated.

Many patients with sepsis develop ARDS with rapid progression to respiratory failure ${ }^{9}$ as in Ezri's report. Infection initiates SIRS by activation of multiple mediators, ${ }^{10}$ often induced by endotoxin. The mediator cascade includes humoral systems such as the complement system, the coagulation/fibrinolytic system, and the 


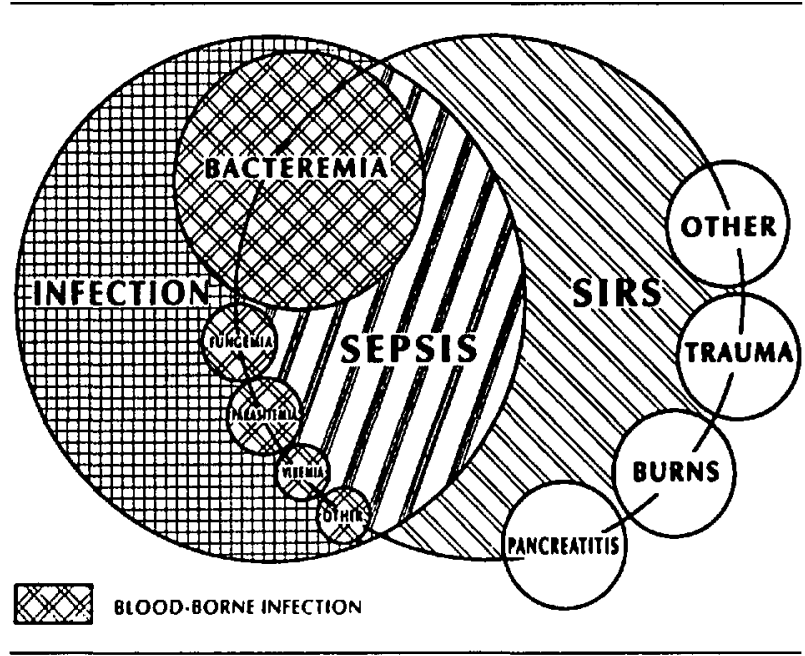

FIGURE Schematic illustrating the interrelationships among systemic inflammatory response syndrome (SIRS), sepsis, bacteremia, infection and other non-infectious conditions. (Reprinted with permission of Critical Care Medicine.)

kallikrein-kinin system as well as cell-based mechanisms including neutrophils, platelets, mast cells, and macrophages. Stimulus multiplication and intensification occurs because cytokines such as tumour necrosis factor (TNF) and interleukin I, released by activated macrophages, interact with these humoral and cellular systems to release or produce proteases, eicosanoids, platelet actuating factor, nitric oxide and reactive $\mathrm{O}_{2}$ products to cause endothelial and epithelial injury or dysfunction. ${ }^{10,11}$ Normally, this system plays a critical role in a normal host's defence; however, concern has been expressed that TNF and interleukin I in sepsis are "good cytokines gone bad."12 In SIRS, there is overproduction of cytokines resulting in uncontrolled and detrimental inflammatory effects, leading to multi-organ dysfunction and death. 10,12

Considering this pathophysiology, ARDS in sepsis can be viewed as the pulmonary manifestation of a systemic disease. ${ }^{13}$ Since the aetiology is multi-factorial, a multifaceted therapeutic approach which interferes with these complex mediator systems has been suggested. The treatment "cocktail"12 could include antibodies to circulating endotoxin, monoclonal antibodies against TNF or recombinant human interleukin I receptor antagonists as well as therapy focused on other pathophysiological factors such as surfactant depletion, ${ }^{14}$ manipulation of nitric oxide synthesis ${ }^{15}$ or the use of nitric oxide to decrease pulmonary hypertension. ${ }^{16}$ Documented effectiveness of these potential pharmacological treatments for ARDS in sepsis is lacking and detailed studies outlining efficacy are now in progress. ${ }^{17}$ Some concern has been expressed that antibody therapy may do more harm than good or that the beneficial effects of these mediators may be neu- tralized. ${ }^{15,18,19}$ Experience with anti-endotoxin monoclonal antibody therapy suggested that treated patients without gram negative sepsis may have a higher mortality than untreated patients. ${ }^{20}$ Presently, there are no rapid tests generally available to determine which patients would benefit or might have adverse effects from cytokine blockade. "Ideally, the development of a rapid sensitive assay for specific serum cytokine/anti-cytokine factors could guide clinical anti-mediator therapy in the future. ${ }^{19}$

These new therapeutic agents are all expensive adjuncts to conventional therapy of sepsis-related ARDS and require randomized, controlled multicentred trials before routine clinical use can be considered. If it is to be meaningful, such "effectiveness research" 17 must differentiate between the results obtained in "ideal circumstances," for example, in centres of excellence conducting randomized clinical trials compared with the use of such agents in routine clinical practice. The former is referred as efficacy whereas the latter is effectiveness. Traditionally, new technologies have been introduced into routine clinical practice without formal testing of effectiveness. The scope of the potential economic problem posed by indiscriminate introduction of unproven pharmacological therapies for ARDS warrants urgent attention. The potential for such treatments to become "budget busters"21 to the health care system has been noted in the United Kingdom ${ }^{21}$ and the United States ${ }^{22}$ where estimated annual costs for anti-endotoxin monoclonal antibody (HA-1A) treatment for sepsis have been $£ 100$ million $^{21}$ and $\$ 1.6$ billion ${ }^{22}$ respectively. Using a multi-hospital database we $\mathrm{e}^{23}$ have recently estimated the annual cost of treating all septic ICU patients in Ontario with one dose of an anti-endotoxin monoclonal antibody ( $\$ 3750$ U.S. per dose). The increased annual cost to the provincial pharmacy budget would be approximately $\$ 9$ million annually (sensitivity analysis ranged from $\$ 6.12$ to $\$ 13.78$ million). This estimate assumed that treatment would be adjunctive and other ICU resource consumption, such as length of stay, would remain stable. Schulmann et al. ${ }^{24}$ conducted a more detailed cost-effectiveness analysis for introduction of this drug in the United States. Surfactant repletion therapy for adults may cost $\$ 10,000-\$ 15,000$ per treatment ${ }^{14}$ and ongoing multi-centre studies are now evaluating its effectiveness for sepsis-related ARDS. This emphasizes the need for properly designed placebocontrolled trials. Such investigations are expensive and may require new, innovative funding mechanisms with collaboration between industry, the health care system and clinicians. However, the cost of such studies will be modest compared with the cost to patients and their health care system if unproved technologies are indiscriminately applied. There is an urgent need to address the issue of technology assessment ${ }^{25}$ within the Cana- 
dian health care system and promote a thoughtful implementation of new, innovative yet expensive techniques for specific groups of patients. Inappropriate utilization of resources and therapy could compound our existing problems funding the care of critically ill patients.

This creates a challenge for clinicians to evaluate which ARDS patient may benefit from a new therapy and determine the optimal time in the disease process to initiate a given treatment. It is important that clinicians understand the pathophysiological basis of the disease process and the advantages and limitations of new forms of therapy. It is likely that an increasing number of anaesthetists will treat patients with sepsis and ARDS in the perioperative period and intensive care unit because sepsis, as a cause of morbidity and mortality, is increasing. ${ }^{6}$ Many of these treatments will be pharmacological. Unlike the mechanical support technologies that anaesthetists have previously used and developed, these pharmacological tools have a greater potential for widespread indiscriminant use. Such technologies as extra-corporeal $\mathrm{CO}_{2}$ removal $^{26}$ and intravenous oxygenation devices (IVOX) ${ }^{27}$ require extensive technical expertise in a specialized setting. These technologies will not be as subject to widespread use as drug therapy. Alternative modes of ventilatory support for ARDS have been used in clinical trials, such as airway pressure release ventilation, inverse ratio ventilation and permissive hypercapnia and may add ventilator acquisition cost to a subset of ARDS patients.

Only by defining the specific disease process and the severity of acute lung injury can clinical investigations clarify the usefulness of new pharmacological therapies and allow the clinician to intervene in a timely and costeffective manner. Anaesthetists in the future will be involved in the care of patients with sepsis and ARDS who are being treated with one or more of these new modalities. Not all cases of sepsis and ARDS resolve as quickly as that in Ezri's report; ${ }^{8}$ therefore, it will be important for the anaesthetist to be aware of the benefits of these therapeutic options in specific subsets of ARDS patients.

\section{ARDS et sepsis}

Le terme ARDS définit un ensemble de troubles entraînant une atteinte pulmonaire aigüe. Cependant, conséquence de différentes terminologies, la définition de l'ARDS manquait de spécificité et entraînait des difficultés pour faire le diagnostic clinique et comparer des investigations cliniques.' Les critères définis au départ dans les premiers $\mathrm{ARDS}^{2}$ comprenaient, la tachypnée, la dyspnée, le degré d'hypoxémie, les infiltrats pulmonaires sur les clichés standards, la réduction de la compliance pulmonaire. A ces critères s'ajoutèrent (Murray et al. ${ }^{\prime}$ ) le stade de la maladie (aiguie ou chronique), l'étiologie du processus sous-jacent et la sévérité de l'atteinte pulmonaire aigüe, l'ensemble regroupé en un score d'atteinte pulmonaire calculé selon l'extension des infiltrats radiographiques, le rapports $\mathrm{PaO}_{2} / \mathrm{FIO}_{2}$, le niveau de PEEP nécessaire pour maintenir le rapport $\mathrm{PaO}_{2} / \mathrm{FIO}_{2}$ et la compliance respiratoire. Cette définition fut un pas décisif dans la mise au point d'une terminologie acceptée et toujours en vigueur après de minines modifications pour la description des cas résents d'ARDS. ${ }^{3-5}$

La terminologie utilisée pour décrire les états septiques fut encore plus changeante et limitée à l'interprétation des investigations cliniques du sepsis. La conférence de consensus de L'American College of Chest Physicians et la Society of Critical Care d'août $1991^{6}$ insistait sur le fait que l'utilisation de diverses définitions pour les termes infection, bactérémie, sepsis, septicémie, syndrome septique, choc septique, de même que l'utilisation du terme syndrome septique pour un état inflammatoire non infectieux entraînait des problèmes pour notamment cọmparer les résultats de différentes études cliniques. Le Comité de la Conférence de Consensus mit au point un terme, le syndrôme de réponse inflammatoire systémique (SRIS) pour décrire une réponse inflammatoire généralisée pouvant être causée par différents facteurs notamment l'endotoxine. ${ }^{6}$ Le SRIS se manifeste par deux ou plus des signes suivants: température $>38^{\circ} \mathrm{C}$ ou $<36^{\circ} \mathrm{C}$; un rythme cardiaque $>90 / \mathrm{mn}$; un rythme respiratoire $>20 / \mathrm{mn}$ ou une $\mathrm{PaCO}_{2}<32 \mathrm{mmHg}(4,3 \mathrm{KPa})$; des globules blancs $>12000 / \mathrm{mm}^{3}$ ou $<4000 / \mathrm{mm}^{3}$ ou plus de $10 \%$ de formes immatures. Le sepsis se définit donc comme la réponse inflammatoire systémique à l'infection qui se manifeste par les signes cliniques du SRIS. Un diagramme schématique de la Conférence de Consensus de l'ACCP/SCCM (Figure) montre comment l'infection, la bactériémie, le sepsis et le SRIS peuvent être reliés l'un à l'autre. La clarification de la terminologie de l'ARDS et du sepsis est nécessaire pour évaluer le devenir de nouvelles approches thérapeutiques des patients atteints de façon critique par un ARDS induit par un sepsis.

Les manifestations pulmonaires de l'ARDS ont de nombreux points communs, quelles que soient les pathologies de départ comme un traumatisme, un sepsis, une inhalation pulmonaire et une pancréatite. La survie des patients dépend de façon claire de la réversibilité du processus pathologique sous-jacent. La thérapeutique est restée empirique, comprenant la ventilation mécanique en pression positive, la PEEP, l'aide au remplacement 
des volumes intravasculaires et au débit cardiaque, ainsi que le traitement de la pathologie de départ. En dépit d'une meilleure compréhension de la physiopathologie, la mortalité de l'ARDS du à un sepsis demeure entre 40 et $70 \%{ }^{7}$

Dans cette édition du Journal, le cas d'un homme de 72 ans qui a développé une détresse respiratoire sévère avec hypoxémie en période post-opératoire immédiate, est décrit par Ezri et al. ${ }^{8}$ Cet ARDS (du à une bactériémie et à un sepsis) a rendu difficile le sevrage ventilatoire après une dissection radicale du cou. L'hypoxémie et l'atteinte pulmonaire aiguie associées régressèrent rapidement sous thérapeutique de support car la cause du sepsis fut supprimée rapidement. Ceci est une expérience clinique commune lorsque la source dinfection réversible est mise à jour et éradiquée.

Beaucoup de patients avec un sepsis développent un ARDS progressant rapidement vers une détresse respiratoire comme dans le cas décrit. ${ }^{9}$ Le sepsis déclenche un SRIS par l'activation de nombreux médiateurs, ${ }^{10}$ souvent induits par l'endotoxine. La cascade des médiateurs comprend des systèmes humoraux tels que le système du complément, le système coagulation/fibrinolyse, le système kallicreïne-kinine ainsi que des mécanismes cellulaires notamment les polynucléaires neutrophiles, les plaquettes, les mastocytes et les macrophages. La multiplication et l'intensification des stimulis surviennent car des cytokines telles que le TNF et l'interleukine 1 libérées par les macrophages activés, interagissent avec ces systèmes humoraux ou cellulaires pour libérer ou produire des protéases, des eicosanoïds, du PAF, de l'oxyde nitrique et des radicaux libres responsables des lésions ou des dysfonctions endothéliales ou épithéliales. ${ }^{10,11}$ Normalement ce système joue un rôle important dans la réponse normale de l'hôte. Cependant, certaines remarques ont été faites concernant le TNF et linterleukine 1 dans le sepsis "bonnes cytokines devenues mauvaises $" .^{12}$ Dans le SRIS, il y a une surproduction de cytokines conduisant à des effets inflammatoires délétères incontrollés, puis à une dysfonction poly-viscérale et à la mort. ${ }^{10,12}$

Considérant donc cette physiopathologie, l'ARDS dans le sepsis peut-être vu comme étant la manifestation pulmonaire d'une maladie systémique. ${ }^{13}$ Etant donné l'étiologie multifactorielle, une approche thérapeutique à diverses facettes qui interfère avec ces systèmes de médiateurs complexes a été suggérée. "Le cocktail" thérapeutique pourrait comprendre des anticorps contre l'endotoxine circulante, des anticorps monoclonaux contre le TNF ou des antagonistes aux récepteurs de l'interleukine 1 humaine recombinée ainsi que des thérapeutique axées sur les autres facteurs physiopathologiques tels que la déplétion du surfactant, ${ }^{14}$ la manipulation de la synthèse de l'oxyde nitrique, ou l'utilisation de l'oxyde nitrique ${ }^{15}$ pour diminuer l'hypertension pulmonaire. ${ }^{16} \mathrm{La}$ preuve de l'efficacité de ces thérapeutiques potentielles dans l'ARDS du sepsis manque, et des études détaillées et documentées sont en cours actuellement. ${ }^{17}$ Quelques remarques ont été faites au sujet notamment de la thérapeutique par les anticorps qui peut-être plus néfaste que bonne ou des effets bénéfiques de ces médiateurs qui peuvent être neutralisés. ${ }^{15,18,19}$. L'utilisation de la thérapeutique par des anticorps mono-clonaux anti-endotoxine tend à montrer que les patients traités, sans sepsis à gram négatif, pourraient avoir une mortalité supérieure par rapport aux patients non traités. ${ }^{20}$ Actuellement, il n'y a pas de tests rapides disponibles permettant de savoir quels patients pourraient bénéficier du blocage des cytokines ou au contraire développer des effets adverses. "Idéalement, la mise au point d'un test rapide et sensible pour les facteurs spécifiques du sérum: cytokine/anti-cytokine pourrait guider la thérapeutique clinique par les antimédiateurs dans l'avenir. ${ }^{18}$

Ces nouveaux agents thérapeutiques sont couteux et nécessitent des études randomisées, controlées et multicentriques avant que leur utilisation clinique de routine soit envisagée. Si cela est important à démontrer, une telle " étude sur l'efficacité " ${ }^{17}$ doit distinguer les résultats obtenus dans des " circonstances idéales", comme par exemple dans des centres ayant conduit des études cliniques randomisées excellentes et ceux obtenus dans des cas de pratiques cliniques courantes. Le premier se réfère à l'efficacité proprement dite alors que le second se réfère à la "productivité ". Traditionellement, les nouvelles technologies deviennent d'utilisation clinique courante sans preuve formelle de leur efficacité. Les aspects du problème économique potentiel posés par l'introduction sans critères discriminatifs de thérapeutiques aux effets pharmacologiques non prouvés dans le cas de l'ARDS imposent une attention urgente. Le risque que ces traitements ne deviennent des "budgetivores " ${ }^{21}$ dans le système de soins a été noté en Angleterre ${ }^{21}$ et aux USA ${ }^{22}$ où les coûts annuels estimés des anticorps monocloraux anti-endotoxine dans le traitement des sepsis a été de 100 millions ${ }^{21}$ de livres et 1.6 milliards de dollars ${ }^{22}$ respectivement. En utilisant récemment les données de plusieurs hôpitaux ${ }^{23}$ nous avons estimé le coût annuel du traitement de tous les patients en soins intensifs pour sepsis, en Ontario, par une dose d'anticorps monoclonaux antiendotoxine (3750,00 \$ US par dose). Le surcoût annuel estimé et incombant au budget de la pharmacie provinciale serait d'environ 9 millions de dollars par an (entre 6.12 et 13.78 millions de dollars par an). Cette estimation suppose que le traitement serait accessoire et, que les autres facteurs de consommation en USI tels que la durée du séjour, demeureraient stables. Schulmann et al. ${ }^{24}$ 
a mené une étude plus détaillée sur le rapport coût efficacité de la mise en pratique de ce produit aux USA. La thérapeutique de remplacement du surfactant chez les adultes risque de coûter de 10000 à 15000 dollars/traitement ${ }^{14}$ et des études en cours, multi-centriques évaluent son efficacité dans les ARDS dus à un sepsis. D'où l'importance d'études correctement élaborées, groupe placebo-groupe contrôle. Ces études sont chères et risquent de nécessiter une collaboration étroite entre l'industrie pharmaceutique, les systèmes de soins et les cliniciens pour recueillir des fonds nouveaux. Cependant ce coût semble bien modeste comparé à celui que risque d'engendrer l'utilisation de techniques d'efficacité non prouvée et appliquées sans discrimination. Il y a un besoin urgent d'adresser les résultats de l'évaluation de ces techniques ${ }^{24}$ à l'intérieur du système de soin canadien et de développer lintroduction bien pensée de nouvelles techniques, innovatrices bien que chères pour des groupes de patients déterminés. Une utilisation inappropriée des ressources et des thérapeutiques risque d'ajouter encore à nos problèmes financiers pour les soins aux patients gravement atteints.

Ceci entraîne une obligation pour les cliniciens d'estimer quel patient atteint d'ARDS pourrait bénéficier d'une nouvelle thérapeutique et du moment optimal où ce traitement serait à débuter dans le cours de la maladie. Il est important que les médecins comprennent les bases physiopathologiques de la maladie ainsi que les avantages et les limites des nouvelles thérapeutiques. Il est probable qu'un nombre croissant d'anesthésistes aura à traiter des patients avec sepsis et ARDS en période per-opératoire et en unité de soins intensifs car le sepsis, augmente comme cause de morbidité et de mortalité. ${ }^{6}$ Beaucoup de ces traitements seront pharmacologiques. A l'inverse des thérapeutiques de support utilisées et développées jusqu'à présent, ces agents pharmacologiques sont à haut risque d'utilisation large et sans discrimination. Certaines techniques telles que l'extraction extracorporelle ${ }^{26}$ de $\mathrm{CO}_{2}$ et des machines d'oxygénation intraveineuxe (IVOX) ${ }^{27}$ nécessitent de couteuses expertises techniques dans un environnement spécialisé. Ces techniques ne seront pas aussi sujettes à une utilisation large que les drogues pharmacologiques. Divers modes de ventilation mécaniques dans l'ARDS ont été utilisés, comme la ventilation en relaxation de pression, la ventilation avec rapport $\mathrm{I} / \mathrm{E}$ inversé et la ventilation avec une hypercapnie autorisée et peuvent augmenter le coût d'acquisition d'un respirateur pour un sous-groupe de patients en ARDS.

En définissant le mécanisme spécifique de la maladie et la sévérité de l'atteinte pulmonaire, les investigations cliniques peuvent éclaircir l'utilité des nouvelles thérapeutiques pharmacologiques et permettre au clinicien d'intervenir au moment optimal, d'une façon coût-efficacité optimale. Les anesthésistes dans l'avenir seront impliqués dans les soins de ces patients en sepsis et ARDS traités par une ou plusieurs de ces nouvelles modalités. Tous les cas de sepsis et d'ARDS ne rentrent pas aussi vite dans l'ordre que celui décrit par Ezri. ${ }^{8}$ Cependant, il sera important que les anesthésistes soient conscients des bénéfices de ces options thérapeutiques dans le cadre spécifique des patients en ARDS.

\section{References}

1 Murray JF, Matthay MA, Luce JM, Flick MR. An expanded definition of the adult respiratory distress syndrome. Am Rev Respir Dis 1988; 138: 720-3.

2 Ashbaugh DG, Bigelow DB, Petty TI, Levine BE. Acute respiratory distress in adults. Lancet 1967; 2: 319-23.

3 Radermacher P, Santak B, Wust HJ, Tarnow J, Falke KJ. Prostacyclin for the treatment of pulmonary hypertension in the Adult Respiratory Distress Syndrome: effects on pulmonary capillary pressure and ventilation-perfusion distributions. Anesthesiology 1990; 72: 238-44.

4 Meduri GU, Belenchia JM, Estes RJ, Wunderink RG, El Torky $M$, Leeper $K V J r$. Fibroproliferative phase of ARDS. Clinical findings and effects of corticosteroids. Chest 1991; 100: 943-52.

5 Luce JM, Montgomery AB, Marks JD, Turner J, Metz $C A$, Murray $J F$. Ineffectiveness of high-dose methylprednisolone in preventing parenchymal lung injury and improving mortality in patients with septic shock. Am Rev Respir Dis 1988; 138: 62-8.

6 ACCP/SCCM Consensus Conference: definitions for sepsis and organ failure and guidelines for the use of innovative therapies in sepsis. Crit Care Med 1992; 20: 864-74.

7 Artigas A, Carlet J, Le Gall JR, Chastang CL, Blanch L, Fernandez $R$. Clinical presentation, prognostic factors and outcome of ARDS in the European Collaborative Study (1985-1987). A preliminary report. In: Zapol WM, Lamaire F (Eds.). Adult Respiratory Distress Syndrome, New York: Marcel Dekker Inc., 1991: 37.

8 Ezri T, Szmuk P. Poria I, Schattner A, Shklar B, Soroker $D$. Adult Respiratory Distress Syndrome after radical neck dissection - an uncommon complication. Can J Anaesth 1993; 40: 658-63.

9 Hyers TM. Adult Respiratory Distress Syndrome: definitions, risk factors, and outcome. In: Zapol WM, Lamaire F (Eds.). Adult Respiratory Distress Syndrome, New York: Marcel Dekker Inc., 1991: 23.

10 Neuhof $H$. Actions and interactions of mediator systems and mediators in the pathogenesis of ARDS and multiorgan failure. Acta Anaesthesiol Scand Suppl 1991; 95 : 7-14.

11 Dinarello CA, Gelfand JA, Wolff $S M$. Anticytokine strategies in the treatment of the systemic inflammatory response syndrome. JAMA 1993; 269: 1829-35.

12 Standiford TJ, Strieter RM. TNF and IL-1 in sepsis; good 
cytokines gone bad (Editorial). J Lab Clin Med 1992; 120: 179-80.

13 Bone RC, Balk R, Slotman G, et al. Adult Respiratory Distress Syndrome. Sequence and importance of development of multiple organ failure. The Prostaglandin $E_{1}$ Study Group. Chest 1992; 101: 320-6.

14 Gragory $G A$. Surfactant replacement for respiratory failure: lessons from the neonate. Anesth Analg 1993; 76: 465-6.

15 Battafarano RJ, Dunn DL. Role of nitric oxide during sepsis (Editorial). Crit Care Med 1992; 20: 1504-5.

16 Pearl RG. Inhaled nitric oxide. The past, the present, and the future. Anesthesiology 1993; 78: 413-6.

17 Goodman C. It's time to rethink health care technology assessment. Int J Technol Assess Health Care 1992; 8: 335-54.

18 St. John RC, Dorinsky PM. Immunologic therapy for ARDS, septic shock and multiple-organ failure. Chest 1993; 103: 932-43.

19 Bone RC. Monoclonal antibodies to tumor necrosis factor in sepsis: help or harm? Crit Care Med 1993; 21: 311-2.

20 Tanio CP. Feldman HI. The HA-1A monoclonal antibody for gram-negative sepsis (Letter). N Engl J Med 1991; 325: 280.

21 Taylor D. Centoxin - birth of a budget buster. BMJ 1991; 302: 1229.

22 Schmidt GA. The HA-1A monoclonal antibody for gramnegative sepsis (Letter). N Engl J Med 1991; 32S(4): 280-1.

23 Byrick RJ, Sibbald WJ, Inman KJ, et al. Estimating the financial impact of anti-endotoxin monoclonal antibody therapy in Ontario. (In preparation)

24 Schulman KA, Glick HA, Rubin H, Eisenberg JM. Costeffectiveness of HA-1A monoclonal antibody for gramnegative sepsis. Economic assessment of a new therapeutic agent. JAMA 1991; 266: 3466-71.

25 Technology Subcommittee of the Working Group on Critical Care Ontario Ministry of Health. The assessment of technology in Ontario's critical care system. Can Med Assoc J 1991; 144: 1613-5.

26 Gattinoni L, Pesenti A, Mascheroni D, et al. Lowfrequency positive-pressure ventilation with extracorporeal $\mathrm{CO}_{2}$ removal in severe acute respiratory failure. JAMA 1986; 256: 881-6.

27 Conrad SA, Eggerstedt JM, Morris VF, Romero MD. Prolonged intracorporeal support of gas exchange with an intravenacaval oxygenator. Chest 1993; 103: 158-61. 